\title{
Síntese, Caracterização e Utilização da peneira molecular SBA-15 como adjuvante em fármaco.
}

\author{
Ana Clara Almeida Santiago;(Graduanda de Ciências Biológicas na Universidade do Estado do \\ Rio Grande do Norte-UERN) \\ Fernando Rodrigo Dantas Fernandes; (Mestrando de Ciências Naturais na Universidade do \\ Rio Grande do Norte-UERN) \\ Anne Gabriella Dias Santos; (Prof.(a) do DQ e PPGCN da Universidade do Estado do Rio \\ Grande do Norte-UERN) \\ J osé Hélio de Araújo Filho.(Prof. do DeCB da Universidade do Estado do Rio Grande do \\ Norte)
}

\begin{abstract}
Resumo:
A utilização de plantas no tratamento de diversas doenças é bastante difundida em todo o mundo. No mundo, principalmente em regiões mais pobres a utilização de tratamentos mais baratos é de suma importância. O tratamento de feridas continua sendo um tema de grande importância médica, particularmente na prática cirúrgica, numa época em que o uso indiscriminado de antimicrobianos tem dificultado o tratamento hospitalar dos pacientes, com repercussão no aparecimento cada vez maior de microorganismos resistentes aos antimicrobianos. Adjuvantes são substancias que possuem diversas funções diante dos fármacos a qual são apresentados, possuem funções como aumentar a solubilidade, suspender, aumentar a viscosidade, diluir, lubrificar, estabilizar, promover fluxo, aglutinar, desintegrar, conservar e modificar a liberação de fármacos. Um dos adjuvantes que vem sendo utilizado em pesquisas farmacêuticas é o SBA-15, pois as mesmas possuem propriedades físico-químicas com grande potencial como adjuvante de mucosas.O presente trabalho tem como objetivo testar o SBA-15 como adjuvante conservando os efeitos dos fármacos nos quais possivelmente serão testados. A amostra de SBA-15 foi sintetizada em meio ácido pelo método hidrotérmico utilizando o P123 como direcionador orgânico e o TEOS como fonte de sílica.A amostra obtida foi caracterizada pelas técnicas DRX, TG/DTG e DSC. O SBA-15 foi adicionado a pomada de forma mecânica até que atingisse uma certa homogeneidade. O material mesoporoso SBA-15 foi sintetizado com sucesso de acordo com a literatura e ao ser misturado a pomada o mesmo adquiriu uma boa textura. Foi realizado um teste onde se foi colocada à pomada pura e com o SBA-15 á uma temperatura de 5 graus ${ }^{\circ} \mathrm{C}$ e observado visualmente a variação de textura. A pomada com o SBA-15 mostrou-se mais resistente.
\end{abstract}

palavras-chave:

Pomada; SBA-15; Adjuvante; Ferida.

Espaço reservado para organização do congresso.:Describe in english briefly what is you paper about.

\section{I NTRODUÇÃO}

A utilização de plantas no tratamento de diversas enfermidades, infecciosas ou não, é, na prática, bastante utilizada (MACIEL \& VIANA, 2005), visto que o Brasil possui sérios problemas de saúde, principalmente na região Nordeste, em razão do baixo poder aquisitivo de grande parte da população local. O alto custo dos remédios torna-os inacessíveis até mesmo nos mais simples casos, como dores agudas e/ou crônicas, febres e gripes. Portanto, o uso popular das plantas medicinais foi o primeiro passo 
para o desenvolvimento de novos agentes terapêuticos de baixo custo para uma região tão carente de recursos financeiros e, por outro lado, tão rica em flora, conquanto reúna milhares de espécies vegetais distintas (RABELO et al., 2003).

O tratamento de feridas continua sendo um tema de grande importância médica, particularmente na prática cirúrgica, numa época em que o uso indiscriminado de antimicrobianos tem dificultado o tratamento hospitalar dos pacientes, com repercussão no aparecimento cada vez maior de microorganismos resistentes aos antimicrobianos (BLASER et al, 2007). A cicatrização de feridas é um processo fisiológico que se inicia com resposta inflamatória caracterizada pelo aumento de fluxo sanguíneo, permeabilidade capilar e migração de leucócitos para a região lesada (COELHO et al, 2001). Sendo assim pesquisas que busquem novos fármacos é de suma importância para indústria farmacêutica. Sabendo que o óleo de Lippia possui efeito cicatrizante, buscaremos potencializar este efeito com o SBA-15. Como lesões podem causar muitos prejuízos à sociedade, principalmente nos membros inferiores impossibilitando o trabalho, pesquisas são realizadas para amenizar estes problemas. $\mathrm{O}$ primeiro passo é a utilização de ervas medicinal, em seguida é adicionada a pomada que não possuem aditivos farmacológicos ervas com efeitos cicatrizantes, analgésicos etc.

As pomadas são um grupo de preparações farmacêuticas muito heterogéneo, caracterizado pela sua consistencia semi-solida. Elas destinam-se a serem aplicadas sobrea peleou membranas mucosas, a fim de exercer ação local ou levar a penetração na pele atingindo outros niveis dermicos (MONTSERRAT, 2007) As pomadas contêm menos de 20\% de água e de voláteis e mais do que $50 \%$ de hidrocarbonetos, ceras, ou polióis, como veículo (LOURENÇO, 2013).

O Creme base utilizado nesta pesquisa foi o Lanette, bastante utilizado em pesquisas. As bases de cremes e loções cremosas mais indicadas são as bases auto- emulsionantes. Entre estas bases, uma aniônica, muito usada e muito antiga é o creme Lanette, preparado com a mundialmente conhecida cera Lanette (álcool cetoestearílico e cetil estearil sulfato de sódio). Esta base é uma das mais utilizadas pela boa estabilidade que apresentam (ZANIN,2001). Vários fármacos inclusive pomadas tem em sua formulação adjuvantes que ajudam no processo de tratamento.

Adjuvantes são substancias que possuem diversas funções diante dos fármacos a qual são apresentados. Possuem funções como aumentar a solubilidade,suspender, aumentar a viscosidade,diluir,lubrificar,estabilizar, promover fluxo, aglutinar, desintegrar, conservar e modificar a liberação de fármacos, colorir, flavonizar, incrementar a dissolução, molhar, formar filmes, incrementar a compactação, favorecer a compressão e correção de características sensoriais do fármaco, proporcionando a obtenção de diversas preparações farmacêuticas (SILVA, 2013)

Um dos adjuvantes quem vem sendo utilizado em pesquisas farmacêuticas é o SBA-15, pois o mesmo possuem propriedades físico-químicas com grande potencial como adjuvante de mucosas (SCARAMUZZI, 2009). Descrita em 1998 a sílica mesoporosa SBA-15 apresenta estrutura hexagonal com poros (3,1-6,4 nm) altamente ordenados e interligados (ZHAO et al.,1998a ; MATOS et al,.2001). O SBA- 15 é normalmente sintetizado em meio fortemente ácido, utilizando o tetraetilortosilicato (TEOS) como fonte de sílica e o copolímero tribloco Pluronic P123 $\left(\mathrm{EO}_{20} \mathrm{PO}_{70} \mathrm{EO}_{20}\right)$ como direcionador orgânico da estrutura (ZHAO et al.,1998 a). Esse material possui estrutura altamente organizada e características interessantes, tais como elevada área superficial, diâmetro de poro ajustável e alta estabilidade térmica e hidrotérmica (FERNANDES et al., 2012), Esses materiais são capazes de interagir com íons, átomos e moléculas (KRESGE et. al) e devido a essas características apresenta vasta aplicabilidade. O SBA-15 está sendo utilizado como adjuvante mais em outras pesquisas como sua utilização em vacinas contra Hepatite A e na imunização via oral contra infecções.

Tendo em vista o potencial de aplicação do SBA-15 como adjuvantes o seguinte trabalho tem como objetivo sintetizar de acordo com a literatura e inserir esse material em fármacos, aumentando assim a área de utilização do SBA-15.

\section{MATERI AL E METODOS}

A pomada utilizada neste experimento foi o creme Lanette,que é comercializado em farmácias de manipulação. O creme não tem nenhum aditivo farmacológico podendo assim receber óleos essenciais e outros produtos provenientes de plantas, no qual se deseja avaliar o potencial seja ele cicatrizante antinflamatorio ou analgésico a nível tópico. 


\subsection{Sínteses do SBA-15:}

A amostra de SBA-15 foi sintetizada pelo método hidrotérmico. O procedimento de síntese foi seguido pelo método proposto por Zhao et. al (1998), utilizando o copolímero tribloco P123 (EO20PO70EO20), ácido clorídrico 37 \%, água destilada e o tetraetilortosilicato (TEOS), seguindo a seguinte proporção molar: 1,000 TEOS: 0,015 P123: 2,750 HCl: 166,0 H2O. O processo de síntese começou com a dissolução do P123 na água destilada e no ácido clorídrico sob agitação e com temperatura entre 35 e $40^{\circ} \mathrm{C}$ por $2 \mathrm{~h}$. Após esse tempo, a fonte de sílica e o TEOS foram adicionados. $\mathrm{O}$ resultado da mistura foi mantido sob agitação e com a mesma temperatura por $24 \mathrm{~h}$. Assim o gel obtido foi transferido para uma autoclave de teflon revestida de ácido inoxidável, o qual foi colocado na estufa a $100^{\circ} \mathrm{C}$ e mantido a mesma temperatura por $48 \mathrm{~h}$. Após esse tempo o material foi resfriado a temperatura ambiente, filtrado a vácuo e lavado com $50 \mathrm{ml}$ de uma solução de $2 \%$ de $\mathrm{HCl}$. O material foi seco a temperatura ambiente por 48 , logo após foi calcinado a $550{ }^{\circ} \mathrm{C}$ por 6 horas.

\subsection{Caracterizações do SBA-15}

Para determinar se a estrutura referente a materiais do tipo SBA- 15 foi obtida com sucesso foi realizada a análise de difração de Raios- X (DRX). As amostras foram analisadas utilizando o equipamento Rigaku modelo Mini Flex II, usando radiações de $\mathrm{CuK} \alpha$, em uma voltagem de $30 \mathrm{k} / \mathrm{V}$ e corrente de tubo de $30 \mathrm{~m}$, onde as amostras foram analisadas em baixo ângulo $(2 \theta)$ de $0,5^{\circ}$ a $5,0^{\circ}$. A análise termogravimétrica (TG/DTG e DSC) foi realizada com o intuito principal de acompanhar as perdas de massa nas amostras para se determinar a melhor temperatura para a calcinação dos materiais com o intuito de remover o direcionador orgânico da estrutura do SBA- 15 já formado. Para isso as amostras foram submetias a um aquecimento de 25 a $900{ }^{\circ} \mathrm{C}$ com um aumento de temperatura de $10^{\circ} / \mathrm{min}$, sob atmosfera de nitrogênio com fluxo de $25 \mathrm{~mL} / \mathrm{min}$. O equipamento que foi utilizado para as análises de TG e DSC foi o modelo STA 449 júpiter da Netzsch.

\subsection{ADI ÇÃO DO SBA-15 A POMADA}

O creme base Lanette foi comprado em uma farmácia de manipulação. O mesmo foi utilizado, pois não possui nenhum aditivo farmacológico que interfira nas pesquisas. $O$ creme base Lanette(pomada) foi adicionado ao SBA-15 de forma mecânica ate que atingisse uma mistura homogêneo. Foi adicionado 10\% de SBA-15 em 5 gramas da pomada.

\subsection{TESTE DE TEMPERATURA}

Foram colocadas duas placas de petri contendo $5 \mathrm{~g}$ da pomada, sendo que uma havia recebido $0,500 \mathrm{~g}$ de SBA-15. Os mesmos foram levados à estufa a 50 graus ${ }^{\circ} \mathrm{C}$, foi observado a variação de consistência e perca de água. Foram feitas observações do aspecto do material por meio do registro das imagens a cada 20 minutos por 1 hora e 20 minutos.

\section{RESULTADOS E DISCUSSÕES}

O respectivo trabalho e seus resultados são testes iniciais utilizando o SBA-15 como adjuvante. O SBA-15 foi adicionado à pomada para que se pudesse observar sua eficácia como adjuvante em fármacos a nível tópico poderão ser feitas posteriormente para confirmar seus feitos

\subsection{FRAÇÃO DE RAIOS-X (DRX)}

O Gráfico 1 apresenta o resultado obtido através da técnica de difração de raios-x para a amostra de SBA-15. 


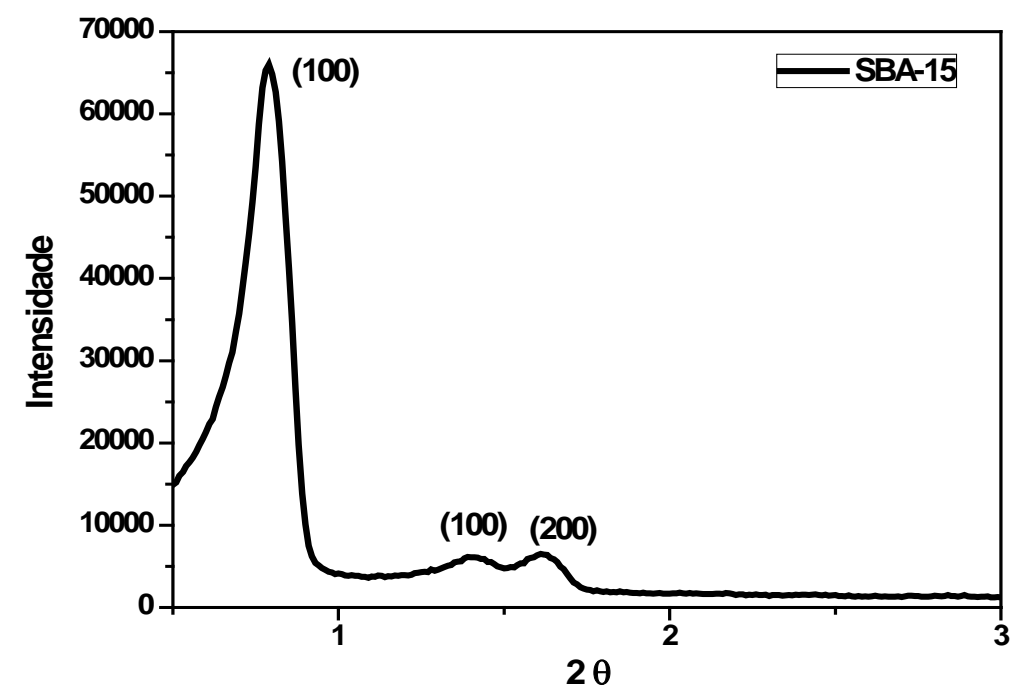

Gráfico 1. Difração de raios-x para a amostra de SBA-15.

Com base nos dados obtidos através da técnica de difração de raios-x, observa-se que a amostra sintetizada apresentou as três reflexões, com índices de Miller (100), (110) e (200), correspondentes ao primeiro pico bastante intenso, o segundo e o terceiro um pouco mais discretos, característicos da estrutura mesoporosa bidimensional com simetria $\mathrm{P} 6 \mathrm{~mm}$, que representam a estrutura hexagonal de materiais bem ordenados do tipo SBA-15 (ZHAO, et al. 1998 b; FERNANDES et al., 2012; JING, et al. 2014). O material se apresentou bem organizado, o que pode ser comprovado pela alta intensidade do seu pico principal (100).

\subsection{ANÁLI SES TERMOGRAVI MÉTRI CAS (TG/ DTG)}

O Gráfico 2 mostra as curvas termogravimétricas da amostra de SBA-15 não calcinada.

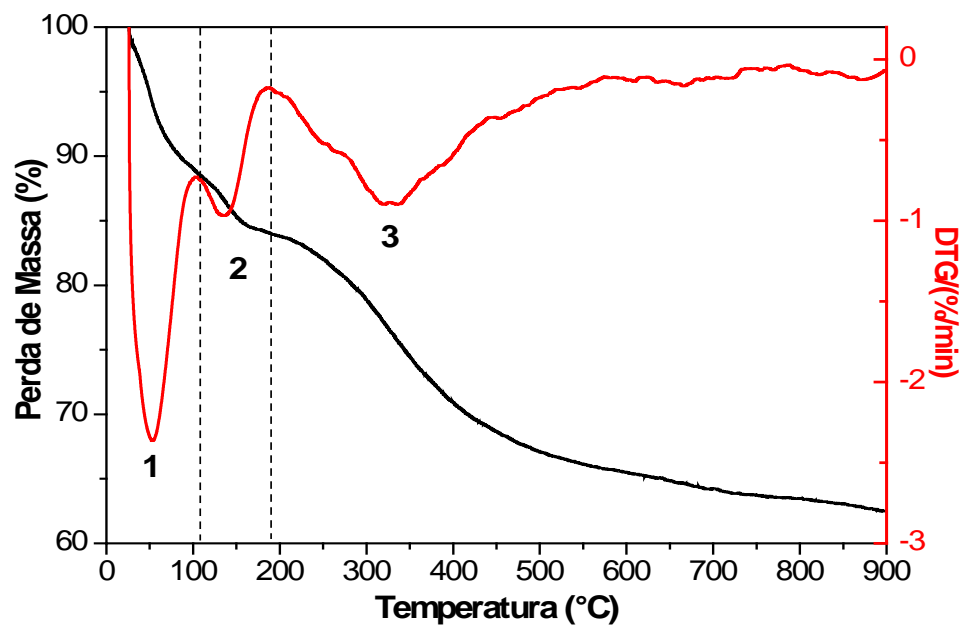

Gráfico 2. Curvas termogravimétricas da amostra de SBA-15 não calcinada.

Pode- se observar através dos resultados obtidos na análise termogravimétrica que a amostra de SBA-15 apresentou três perdas de massa referentes respectivamente, a saída da água fisissorvida no material, a degradação do direcionador orgânico e a condensação de grupos silanóis ainda presentes no material (FERNANDES et al., 2012). Através da análise termogravimétrica determinou-se a temperatura de calcinação em $550^{\circ} \mathrm{C}$, pois nessa temperatura já não existe nenhuma perda de massa referente ao direcionador orgânico. 
Os Gráficos 3 e 4 mostram os resultados da TG e DTG, respectivamente, das amostras de SBA-15, Pomada e Pomada com SBA-15.

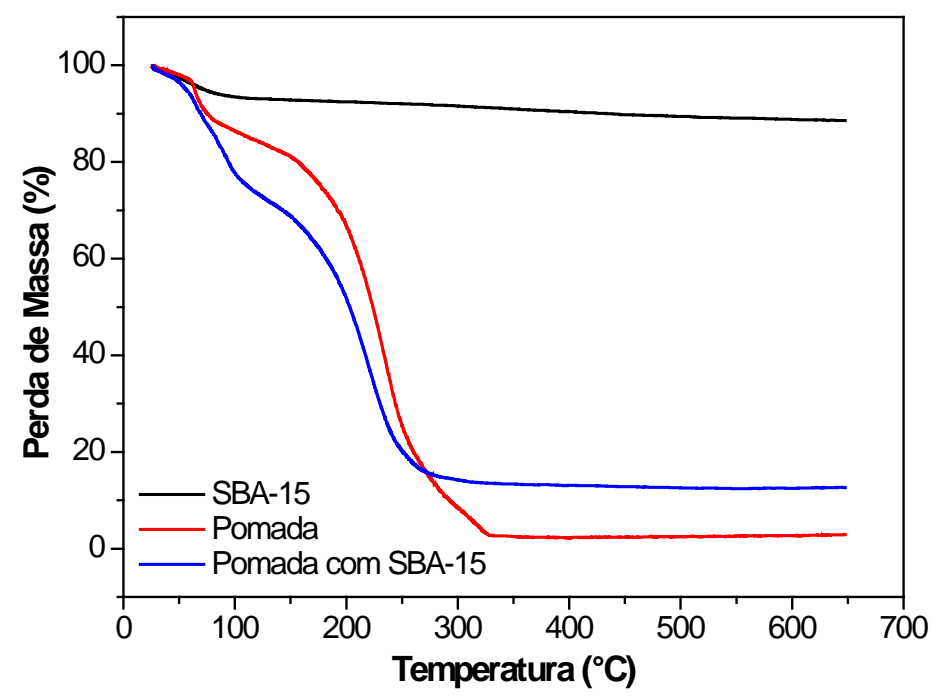

Gráfico 3. Curvas da TG das amostras de SBA-15, Pomada e Pomada com SBA-15.

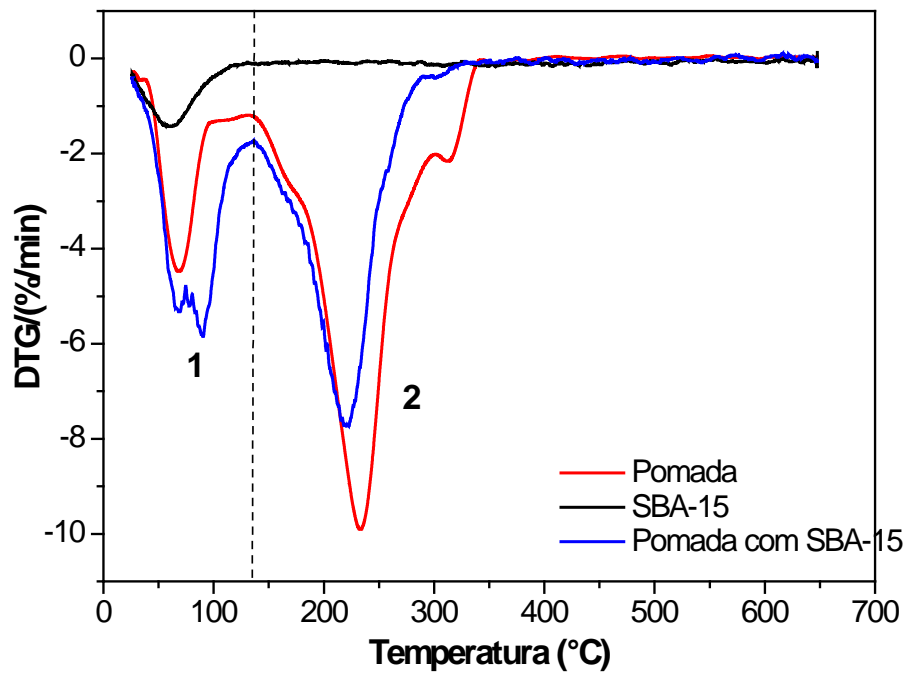

Gráfico 4. Curvas da DTG das amostras de SBA-15, Pomada e Pomada com SBA-15.

Com base nos dados da TG e DTG das amostras analisadas, pode- se observar que a amostra de SBA-15 apresentou apenas uma perda de massa, que é atribuída a saída da água fisissorvida do material. Para as amostras da pomada e da pomada com SBA-15, pôde-se observar duas faixas onde se ocorre perda de massa. A primeira é atribuída a água presente nas amostras e a segunda é atribuída a degradação da pomada. Pode- se perceber que a amostra de pomada com SBA-15 apresentou a faixa saída da água presente no material, em temperaturas mais elevadas do que somente a amostra de pomada pura. Isso significa que a amostra de pomada com SBA-15 perde água com mais dificuldade o que é considerado um resultado importante, haja visto que a água é um dos fatores mais importantes para a eficácia do fármaco., Esse fato corrobora com os resultados encontrados no teste de temperatura realizado nas amostras e que será descrito na seção 3.3. Com relação a degradação da pomada não houve diferença de temperatura significativa entre as amostras. 


\subsection{AVALI AÇÃO DA ESTABI LI DADE TERMI CA DA POMADA}

A Figura 1 mostra o aspecto da pomada antes e após a adição do SBA-15.

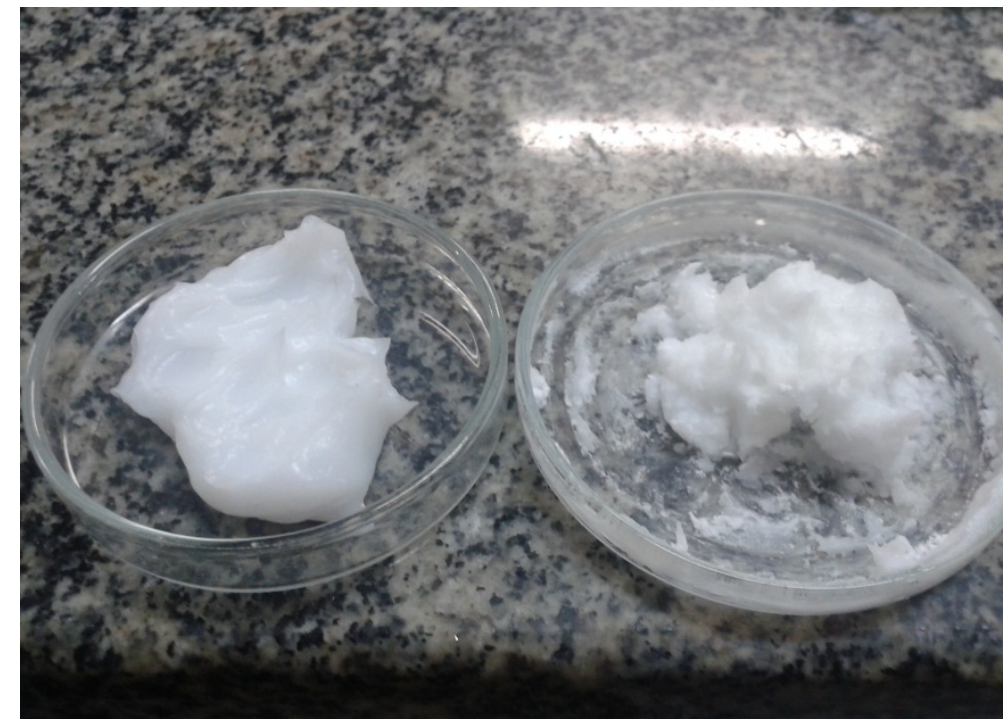

Figura 1. Diferença no aspecto da pomada pura(ESQUERDA) e com SBA-15(DIREITA). ARQUIVO PESSOAL.

Neste trabalho o SBA-15 foi adicionado a uma pomada onde a mesma adquiriu consistência mais aderente, como pode ser observado na Figura 1. Essa mudança na aderência pode indicar melhores resultados nos tratamentos de pomadas com adição do SBA-15, já que esta permaneceria mais tempo em contato com o lugar lesionado, diminuído o tempo de cicatrização.

\section{TESTE DE TEMPERATURA}
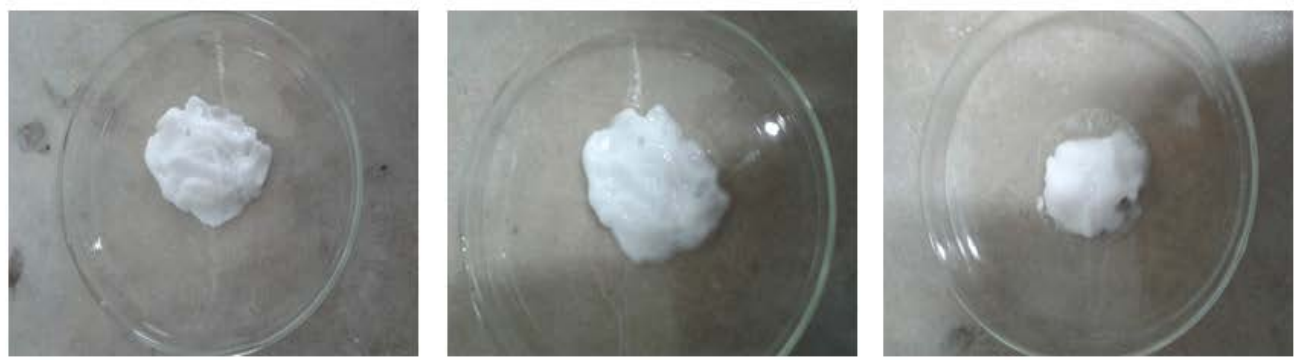

A

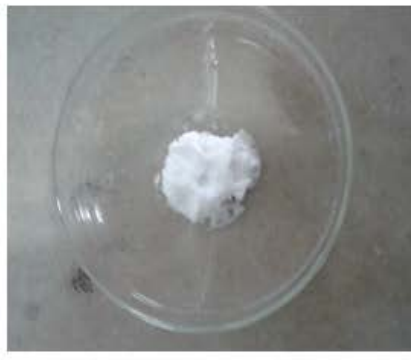

20 MINUTOS

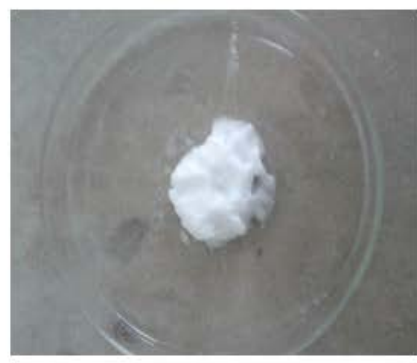

40 MINUTOS

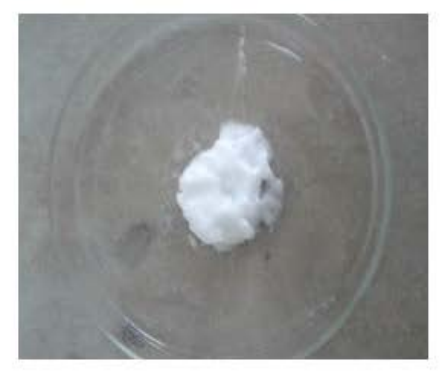

80 MINUTOS

Figura 2. Comparação visual do teste de temperatura entre a pomada pura (A) e a pomada com SBA15(B).ARQUIVO PESSOAL.

Um teste rápido levando em consideração a temperatura mostrou visualmente que a pomada com adição do SBA-15 não perde tanta água em exposição a temperatura testada em relação a pomada pura,. Como pode ser visto na Figura 3 A pomada pura resseca a ponto de tornar-se uma cera, perdendo 
assim sua capacidade como adjuvante, (Figura 3). A pomada com a adição do SBA-15 não teve alterações visíveis que possam comprovar a olho nu que a mesma perdeu sua eficácia. A partir disto pode pressupor que a pomada com o SBA-15 torna-se mais eficiente, pois a água é um dos adjuvantes universais (VALE, 2006), que permitem que o fármaco tenha maior solubilidade, lubrificação e no caso lesões a água deixa a crosta formada elo sistema imune mais susceptível infiltração do fármaco na pele (no caso a pomada) melhorando assim os resultados. Esse fato corrobora com os resultados encontrados nas análises termogravimétricas realizadas nas amostras.

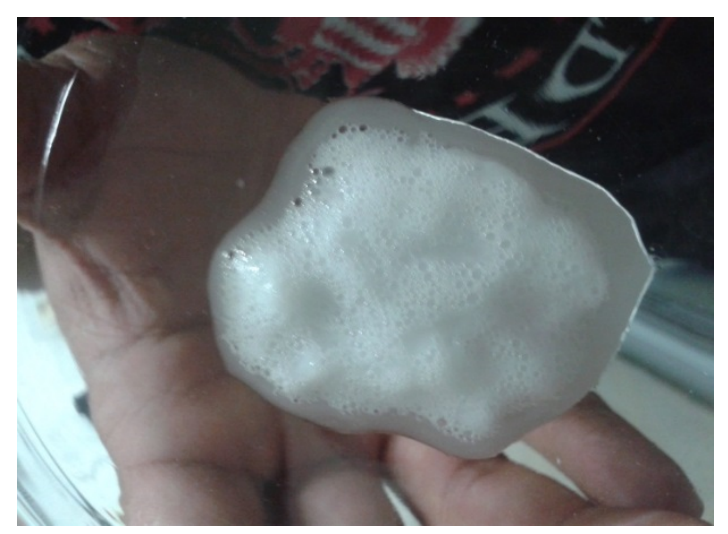

Figura 3. Pomada pura ressecada após o teste de temperatura.ARQUIVO PESSOAL.

\section{CONCLUSÕES}

O SBA-15 foi sintetizado com êxito, de acordo com os resultados de DRX comparados com a literatura. Com base em testes simples o SBA-15 reagiu bem à presença da pomada podendo assim se tornar um adjuvante de fármaco utilizado na indústria farmacêutica ampliando sua área de utilização em feridas infectadas. A adição do SBA-15 na pomada melhorou aspectos físicos da mesma e ajudou na preservação da água nas amostra o que é bastante significativo devido a importância da água na eficácia do fármaco. Contudo há a necessidade da realização de uma pesquisas mais detalhada sobre o assunto em questão.

\section{Synthesis, Characterization and Using the SBA -15 molecular sieve as a drug adjuvant}

\section{Abstract:}

The use of plants in the treatment of various diseases is widespread throughout the world. In the world, especially in poorer regions the use of cheaper treatments is of paramount importance. The wound care remains a major medical important issue, particularly in surgical practice, at a time when the indiscriminate use of antibiotics has hampered hospital treatment of patients, with repercussions on the increasing emergence of antimicrobial-resistant microorganisms. Adjuvants are substances that have various functions before the drugs which they are presented. Have functions as increasing solubility, suspend, increase viscosity, dilute, lubricate, stabilize, promote flow, coalesce, disintegrate, save and modify the release of drugs, one of the builders who has been used in pharmaceutical research is the SBA-15, since the same physical-chemical properties with great potential as mucosal adjuvant. This study aims to test the SBA-15 as adjuvants, preserving the effects of the drugs in which are likely to be tested. The sample of SBA-15 was synthesized in an acid medium by hydrothermal method Using the 
P123 as organic driver and TEOS as a source of silica. The sample obtained was characterized by XRD techniques, TG / DTG and DSC.O SBA-15 was added to mechanically ointment until it reached a certain homogeneity. The mesoporous materials SBA-15 was successfully synthesized according to literature to be mixed with the same ointment acquired a good texture. A test was performed where the pure ointment was placed and the SBA-15 at a temperature 5 degrees $\mathrm{C}^{\circ}$ and observed visually the change of texture. The ointment with SBA-15 was more resistant.

Keywords: Pomade; SBA-15; Adjuvant; Wounds.

\section{Referências bibliográficas}

BLASER,G et al. Effect of medical honey on wounds colonised or infected with MRSA. J Wound Care.; v.16, p.325-328,2007.

COELHO, COC et al.Biopolímero produzido a partir da cana-de-açúcar para cicatrização cutânea. Acta Cir. Bras, v.17, 2001.

FERNANDES,F. R.D. et al. Síntese e caracterização do sba-15 suportado com Nb2O5. Química: Ciência, Tecnologia e Sociedade. v.1, p.30-40, 2012.

KRESGE, C.T; LEONOWIEZ, M.E. ROTH W.J.;VARTULI J.C.;BECK J.S. Ordered mesopouros molecular sieves synthesized by a liquid-crystal template mechanism.Nature, v 359, p 710-712,1992

LOURENÇO, A. R.N: Administração Tópica de Fármacos - Das restrições aos desafios. Dissertação(Mestrado Integrado em Ciências Farmacêuticas) Universidade Lusófona de Humanidades e Tecnologias. Lisboa, 2013

MACIEL, M. A. M.; PINTO, A. C.; VEIGA JR, V. F. Plantas Medicinais: A Necessidade de Estudos Multidisciplinares. Química Nova, Rio de Janeiro, v. 25, n. 3, p. 429-438, 2002.

MONTSERRAT, T. M.M; Formas Farmacêuticas Semisólidas: POMADAS. Disponível em:http://depa.fquim.unam.mx/amyd/archivero/Pomadas_1441.pdf. Acesso em 25/09/2015

RABELO, E. F. Larvicidal activity of the essential oil from Lippia sidoides Cham. against Aedes aegypti Linn. Memórias do Instituto Oswaldo Cruz, v. 98, n. 4, p. 569-571, 2003.

SCARAMUZZI, K. Efeito adjuvante da Silica Mesoporosa Nanoestruturada SBA-15 na Imunização Pela via Oral.Dissertação (Mestrado em Imunologia). Universidade de São Paulo, São Paulo, 20

SILVA, D, C. Ensaios físicos dos excipientes e avaliação das farmacopeias.Monografia (especialização). Instituto de Tecnologia em Fármacos. Rio de Janeiro. 2013

VALE N, B. Analgesia Adjuvante e Alternativa. Rev. Bras Anestesiologia. v 56, p 530-555, 2006.

ZANIN, S.M. W, et al. Parâmetros físicos no estudo da estabilidade das emulsões. Revista Visão Acadêmica, v. 2, n. 2, p. 47-58, 2001.

ZHAO, D. FENG, J.;HUO,Q.;MELOSH,N.;FREDRICKSON, G.H.;CHMELKA,BF.;STUCKY, G.D.Triblock copolymer syntheses of mesoporous silica with periodic 50 to 300 angstrom pores .Science,v 279, p 548-552, 1998. 